\title{
A new line associated with the triangle
}

\author{
Mowaffaq Hajja and Margarita Spirova \\ Mowaffaq Hajja received his Ph.D. at Purdue University (Indiana, U.S.A.) in 1978. \\ Since then he holds a position at Yarmouk University in Irbid (Jordan) where he is \\ currently a professor. His interests comprise mathematics in general, more specifically \\ elementary geometry. \\ Margarita Spirova received her doctorate degree from the University of Sofia (Bul- \\ garia) in 2005. Presently, she holds an assistant professorship at the same university. \\ Her research interests comprise various aspects of geometry.
}

Let $A B C$ be any triangle in the Euclidean plane $\mathbb{E}$ with side-lengths $a, b$, and $c$, and with angles $A, B$, and $C$, in the standard order. Let $X, Y$, and $Z$ be points on the sidelines $B C$, $C A$, and $A B$, respectively. A fairly well-known theorem of Ceva states that the cevians $A X, B Y$, and $C Z$ are concurrent if and only if the cevian relation

$$
(B X)(C Y)(A Z)=-(C X)(A Y)(B Z)
$$

holds, where $U V$ denotes the directed distance between the points $U$ and $V$. Here, a cevian is a line that joins a vertex to a point on the opposite side. Less known is the fact that the perpendiculars erected from the points $X, Y$, and $Z$ on their respective sides, as

Fällt man von einem Punkt $P$ aus die Lote auf die drei Seiten eines Dreiecks $A B C$, so bestimmen die Fusspunkte $X, Y, Z$ auf diesen Seiten die entsprechenden Seitenabschnitte $\overline{B X}, \overline{C Y}, \overline{A Z}$. Es stellt sich heraus, dass der geometrische Ort aller Punkte $P$, für welche diese Seitenabschnitte sich als Linearformen der Längen $a, b, c$ der Dreiecksseiten ausdrücken lassen, die Gerade durch die Zentren $\mathcal{I}$ und $\mathcal{O}$ des In- bzw. des Umkreises ist. Dies ergänzt die Ergebnisse, dass die Gerade durch $\mathcal{I}$ und den Schwerpunkt $\mathcal{G}$ des Dreiecks der geometrische Ort der Punkte ist, deren baryzentrische Koordinaten projektiv linear in $a, b, c$ sind und dass die Eulergerade durch $\mathcal{O}$ und $\mathcal{G}$ der geometrische Ort der Punkte ist, deren baryzentrische Koordinaten projektiv linear in $\tan A, \tan B, \tan C$ sind. Ausserdem generieren die Autoren durch Untersuchung der Geraden durch $\mathcal{I}, \mathcal{O}$ und $\mathcal{G}$ zusätzliche spezielle Punkte des Dreiecks $A B C$, die im Kimberling-Katalog der „Dreieckszentren“ nicht aufgeführt sind. 
shown in Fig. 1, are concurrent if and only if the condition

$$
(B X)^{2}+(C Y)^{2}+(A Z)^{2}=(C X)^{2}+(A Y)^{2}+(B Z)^{2}
$$

holds; see, for example, [13] and [7, Theorem 6.3.1, p. 96].

Following [22], a center or a center function is defined to be a mapping that assigns to every non-degenerate triangle in the Euclidean plane $\mathbb{E}$ a point in $\mathbb{E}$ in a manner that is symmetric and that respects isometries and dilations. More precisely, a center function $\mathcal{Z}$ satisfies the following properties:

(i) $\mathcal{Z}(\sigma(A), \sigma(B), \sigma(C))=\mathcal{Z}(A, B, C)$ for all permutations $\sigma$ on $\{A, B, C\}$.

(ii) $\mathcal{Z}(\varphi(A), \varphi(B), \varphi(C))=\varphi(\mathcal{Z}(A, B, C))$ for all isometries $\varphi$ of $\mathbb{E}$.

(iii) $\mathcal{Z}(\lambda A, \lambda B, \lambda C)=\lambda \mathcal{Z}(A, B, C)$ for all real numbers $\lambda$.

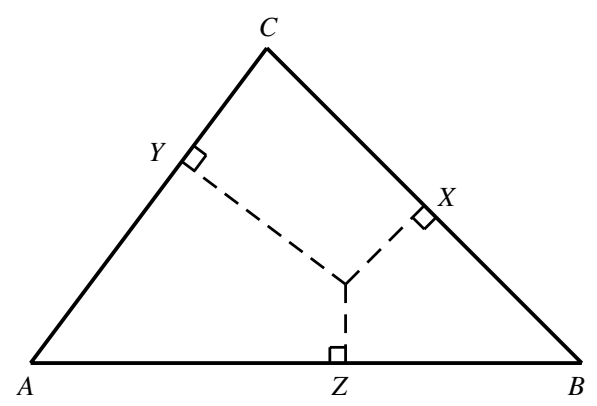

Fig. 1

Let $P$ be a center of triangle $A B C$, and let $X, Y$, and $Z$ be the orthogonal projections of $P$ on the sidelines $B C, C A$, and $A B$, respectively. If the lengths $B X, C Y$, and $A Z$ are linear forms in $a, b$, and $c$, then it follows fairly easily from properties of center functions that the directed lengths of the segments $B X$, etc., are given by

$$
\begin{aligned}
& B X=\frac{a}{2}+t(b-c), C Y=\frac{b}{2}+t(c-a), A Z=\frac{c}{2}+t(a-b) \\
& C X=\frac{a}{2}-t(b-c), A Y=\frac{b}{2}-t(c-a), B Z=\frac{c}{2}-t(a-b)
\end{aligned}
$$

for some $t \in \mathbb{R}$; see the proof of [1, Theorem 1]. Plugging these values in (2), we obtain what, unexpectedly, turns out to be an identity that holds for all $t$; namely the identity

$$
\begin{aligned}
& \left(\frac{a}{2}+t(b-c)\right)^{2}+\left(\frac{b}{2}+t(c-a)\right)^{2}+\left(\frac{c}{2}+t(a-b)\right)^{2} \\
= & \left(\frac{a}{2}-t(b-c)\right)^{2}+\left(\frac{b}{2}-t(c-a)\right)^{2}+\left(\frac{c}{2}-t(a-b)\right)^{2} .
\end{aligned}
$$


Thus every $t \in \mathbb{R}$ defines a center $P_{t}$ in the manner described. Specifically, given $t \in \mathbb{R}$, we locate the points $X, Y$, and $Z$ on the sidelines according to (3) and (4), and we let $P_{t}$ be the point of concurrence of the perpendiculars erected from these points on the respective sidelines.

The centers $P_{t}$ just defined were investigated in [3, Section 9]. There, the authors noted that the centers corresponding to the values $t=0, t=-1 / 2$ and $t=1 / 2$ are the circumcenter, the incenter, and what is referred to in [22] as the Bevan point, and they raised the following question:

Question. What is the curve that the centers $P_{t}$ trace as $t$ varies?

In the theorem below, we answer this question and we prove that the curve in question is, amazingly, a straight line. We also consider those centers for which the angles of $X Y Z$ are linear forms in the angles of $A B C$.

Before stating and proving the main theorem, we remind the reader that the Gergonne (respectively, Nagel) center of triangle $A B C$ is the point of concurrence of the cevians $A A^{\prime}, B B^{\prime}$, and $C C^{\prime}$, where $A^{\prime}, B^{\prime}, C^{\prime}$ are the points where the incircle (respectively, the excircles) touch(es) the sides of $A B C$. That such cevians are concurrent follows from the cevian condition (1). We also point out that the cevian analogue to the above question has an extremely different answer. Specifically, it is shown in [1, Theorem 1] that the only centers of $A B C$ through which the cevians $A A^{\prime}, B B^{\prime}, C C^{\prime}$ are such that $B A^{\prime}, C B^{\prime}$, and $A C^{\prime}$ are linear forms in $a, b$, and $c$ are the centroid, the Gergonne center, and the Nagel center. This heavy contrast stems from the fact that the cevian condition (1) takes the form

$$
\begin{aligned}
& \left(\frac{a}{2}+t(b-c)\right)\left(\frac{b}{2}+t(c-a)\right)\left(\frac{c}{2}+t(a-b)\right) \\
= & \left(\frac{a}{2}-t(b-c)\right)\left(\frac{b}{2}-t(c-a)\right)\left(\frac{c}{2}-t(a-b)\right)
\end{aligned}
$$

and has three solutions only, namely $t=-1 / 2, t=0$, and $t=1 / 2$. Similarly, the only centers for which $\angle B A A^{\prime}, \angle C B B^{\prime}$, and $\angle A C C^{\prime}$ are linear forms in $A, B$, and $C$ are the circumcenter, the incenter, and the orthocenter, and the only centers for which $\angle A C^{\prime} B^{\prime}$, $\angle B A^{\prime} C^{\prime}$, and $\angle C B^{\prime} A^{\prime}$ are linear in $A, B$, and $C$ are the centroid, the orthocenter, and the Nagel center; see [1, Theorem 2] and [2, Theorem 7]. Similar issues are addressed in [17] and [4].

Theorem. Let $A B C$ be a non-degenerate triangle with side-lengths $a, b$, and $c$ in the standard order. For a point $P$ in the plane of $A B C$, let $X, Y$, and $Z$ be the projections of $P$ on the sides $B C, C A$, and $A B$, respectively. Then the centers for which $B X, C Y$, and $A Z$ are linear forms in $a, b$, and $c$ form the straight line that joins the circumcenter and the incenter.

Proof. As seen earlier, the centers in question are precisely the centers $P=P_{t}$ whose projections $X=X_{t}, Y=Y_{t}$, and $Z=Z_{t}$ on the sides $B C, C A$, and $A B$ satisfy (3) and (4) for some $t$. 
Using the lengths of $X B=X_{t} B$ and $X C=X_{t} C$ as given in (3) and (4), we write $X_{t}$ as a convex combination

$$
X_{t}=\frac{1}{a}\left[\left(\frac{a}{2}+t(b-c)\right) C+\left(\frac{a}{2}-t(b-c)\right) B\right]
$$

of the points $B$ and $C$. Multiplying by $2 a$ and simplifying, we obtain

$$
2 a X_{t}=a(C+B)+2 t(b-c)(C-B) .
$$

Since $P_{t}-X_{t}$ is perpendicular to $C-B$, it follows that $P_{t} \cdot(C-B)=X_{t} \cdot(C-B)$. Here, we have treated points in the plane as position vectors and we have used the ordinary scalar product. Without loss in generality, we shall assume that the circumcenter $P_{0}$ of $A B C$ lies at the origin $O$ and that its circumradius is 1 . Then

$$
\begin{aligned}
2 a P_{t} \cdot(C-B) & =2 a X_{t} \cdot(C-B) \\
& =(a(C+B)+2 t(b-c)(C-B)) \cdot(C-B), \text { by }(5) \\
& =a(C \cdot C-B \cdot B)+2 t(b-c)(C-B) \cdot(C-B) \\
& =2 t(b-c) a^{2}, \text { because }\|C\|=\|B\|=1 \text { and }\|C-B\|=a .
\end{aligned}
$$

From this and symmetry, we conclude that

$$
P_{t} \cdot(C-B)=t a(b-c), P_{t} \cdot(A-C)=t b(c-a), P_{t} \cdot(B-A)=t c(a-b) .
$$

We shall now compute the distance $\left\|P_{t}-P_{0}\right\|=\left\|P_{t}\right\|$ between $P_{t}$ and the circumcenter $P_{0}$ and see that it is a constant multiple of $|t|$.

To compute $\left\|P_{t}\right\|$, we let $D=\left[\begin{array}{lll}P_{t} & C-B & A-C\end{array}\right]$ be the $2 \times 3$ matrix whose columns represent the coordinates of the vectors $P_{t}, C-B$, and $A-C$ (with respect to some basis), and we consider the $3 \times 3$ matrix $D^{T} D$, where $D^{T}$ denotes the transpose of $D$. Since $\operatorname{rank}(D) \leq 2$ (in fact $=2$, since $C-B$ and $A-C$ are linearly independent), it follows from the general fact $\operatorname{rank}(U V) \leq \operatorname{rank}(U)$ that $\operatorname{rank}\left(D^{T} D\right) \leq 2$ and that $\operatorname{det}\left(D^{T} D\right)=0$. Therefore

$$
\begin{aligned}
& 0=\operatorname{det}\left(D^{T} D\right) \\
& =\operatorname{det}\left(\left[\begin{array}{ccc}
P_{t} & C-B & A-C
\end{array}\right]^{T}\left[\begin{array}{lll}
P_{t} & C-B & A-C
\end{array}\right]\right) \\
& =\operatorname{det}\left[\begin{array}{ccc}
P_{t} \cdot P_{t} & P_{t} \cdot(C-B) & P_{t} \cdot(A-C) \\
P_{t} \cdot(C-B) & (C-B) \cdot(C-B) & (C-B) \cdot(A-C) \\
P_{t} \cdot(A-C) & (A-C) \cdot(C-B) & (A-C) \cdot(A-C)
\end{array}\right] \\
& =\left|\begin{array}{ccc}
P_{t} \cdot P_{t} & t a(b-c) & t b(c-a) \\
t a(b-c) & a^{2} & Q \\
t b(c-a) & Q & b^{2}
\end{array}\right| \text {, by (6) and where } Q=(C-B) \cdot(A-C) \\
& =\left\|P_{t}\right\|^{2}\left|\begin{array}{cc}
a^{2} & Q \\
Q & b^{2}
\end{array}\right|-t a(b-c)\left|\begin{array}{cc}
t a(b-c) & Q \\
t b(c-a) & b^{2}
\end{array}\right|+t b(c-a)\left|\begin{array}{cc}
t a(b-c) & a^{2} \\
t b(c-a) & Q
\end{array}\right| \\
& =\left\|P_{t}\right\|^{2}\left|\begin{array}{cc}
a^{2} & Q \\
Q & b^{2}
\end{array}\right|-t^{2} a(b-c)\left|\begin{array}{cc}
a(b-c) & Q \\
b(c-a) & b^{2}
\end{array}\right|+t^{2} b(c-a)\left|\begin{array}{cc}
a(b-c) & a^{2} \\
b(c-a) & Q
\end{array}\right| .
\end{aligned}
$$


The right-hand side has the form $\left\|P_{t}\right\|^{2} G-t^{2} H$, where $G$ and $H$ do not depend on $t$. Therefore $\left\|P_{t}\right\|^{2}=t^{2} F$, where $F$ does not depend on $t$, and hence the distance between $P_{t}$ and $P_{0}=0$ is a constant multiple of $|t|$. Since the length of the projection of the segment $P_{t} P_{0}$ on $B C$ is also a constant multiple of $|t|$, being nothing but $|t||b-c|$, we conclude that the absolute value of the slope of $P_{t} P_{0}$, relative to $B C$, is independent of $t$. It follows that the slope of $P_{t} P_{0}$ is independent of $t$. In fact, if $b>c>a$, then $X_{t}$ moves towards $C$ and $Y_{t}$ towards $A$, showing that the slope is positive and hence does not change sign. Thus $P_{t}$ moves on a straight line, necessarily the line joining the circumcenter $P_{0}$ and the incenter $P_{-1 / 2}$.

Remarks. (i) To find a formula for $\left\|P_{t}\right\|$, one uses the Law of Cosines to obtain

$$
2 Q=2(C-B) \cdot(A-C)=-2 a b \cos C=c^{2}-a^{2}-b^{2},
$$

and then plugs this in the determinant above. The result should of course be multiplied by the circumradius $R$ to make up for our assumption that $R=1$. In view of Euler's formula $d^{2}=R(R-2 r)$, where $R$ is the circumradius, $r$ the inradius, and $d$ the distance between the circumcenter and the incenter, one expects to get

$$
\left\|P_{t}\right\|^{2}=4 t^{2}\left\|P_{-1 / 2}\right\|^{2}=4 t^{2} d^{2}=4 t^{2} R(R-2 r) .
$$

(ii) Letting $X, Y$, and $Z$ be as in the theorem, one may consider those centers for which the angles of the pedal triangle $X Y Z$ are linear forms in $A, B$, and $C$. Since

$$
\angle Z X Y=\angle Z X P+\angle Y X P=\angle Z B P+\angle Y C P=\angle B P C-A,
$$

it follows that these are precisely the centers $P$ for which the angles $\angle B P C, \angle C P A$, and $\angle A P B$ are linear forms in $A, B$, and $C$. These centers are the subject of study in [17], where the curve they trace is called the Balaton curve and where the complex behaviour of this curve is fully described.

(iii) Let the centroid, the circumcenter, and the incenter of $A B C$ be denoted, respectively, by $\mathcal{G}, \mathcal{O}$, and $\mathcal{I}$. It is shown in [4] that the locus of the centers of $A B C$ whose barycentric coordinates are projective linear functions in $a, b$, and $c$ is the straight line $L(\mathcal{G}, \mathcal{I})$. It is also noted there that the Euler line $L(\mathcal{G}, \mathcal{O})$ is the locus of the centers of $A B C$ whose barycentric coordinates are projective linear functions in $\tan A, \tan B$, and $\tan C$. It follows that

$$
\begin{aligned}
L(\mathcal{G}, \mathcal{I}) \| B C & \Longleftrightarrow 2 a=b+c, \\
L(\mathcal{G}, \mathcal{O}) \| B C & \Longleftrightarrow 2 \tan A=\tan B+\tan C .
\end{aligned}
$$

A condition for $L(\mathcal{O}, \mathcal{I})$ to be parallel to $B C$ can be found by setting the slope of $L(\mathcal{O}, \mathcal{I})$ equal to 0 . More directly, we let $r$ and $R$ be the inradius and circumradius of $A B C$, and 
we see that

$L(\mathcal{O}, \mathcal{I}) \| B C \quad \Longleftrightarrow \quad$ the areas of triangles $\mathcal{O} B C$ and $\mathcal{I} B C$ are equal

$\Longleftrightarrow \frac{r a}{2}=\frac{R^{2} \sin 2 A}{2}$

$\Longleftrightarrow r a=2 R^{2} \sin A \cos A$

$\Longleftrightarrow \frac{r}{R}=\cos A$, because $2 R=\frac{a}{\sin A}$

$\Longleftrightarrow \cos A+\cos B+\cos C-1=\cos A$, by Carnot's formula [24]

$\Longleftrightarrow \cos B+\cos C=1$.

Thus we have proved that

$$
L(\mathcal{O}, \mathcal{I}) \| B C \Longleftrightarrow \cos B+\cos C=1 .
$$

For earlier references related to (7), see [20, Problem 82, p. 209], [8], [10], [14], [25], and [12]. For (8), see [15, Problem 9, p. 18], [11], and [6], where [6] also describes the locus of $A$ when $A B C$ is a triangle having a fixed base $B C$ and satisfying $2 \tan A=\tan B+\tan C$. For (9), see [26] and [19], where [19, Corollary 4] gives another geometric characterization of the condition $L(\mathcal{O}, \mathcal{I}) \| B C$.

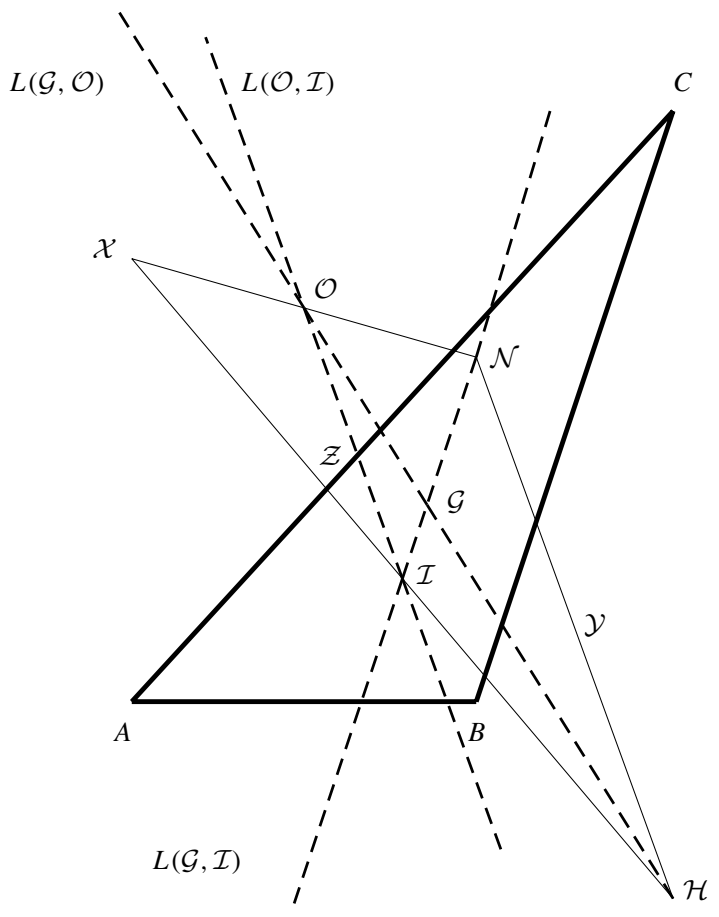

Fig. 2 
(iv) In [28, Exercise 5.7], the area of the triangle $\mathcal{G O I}$ is given by the elegant formula

$$
[\mathcal{G O I}]=\left|\frac{s(b-c)(c-a)(a-b)}{24 K}\right|,
$$

where $s$ is the semiperimeter and $K$ the area of $A B C$. An equivalent formula is given in [5, Section 4.4, pp. 105-106]. It follows that two (and hence all) of the three lines $L(\mathcal{G}, \mathcal{O})$, $L(\mathcal{G}, \mathcal{I})$, and $L(\mathcal{O}, \mathcal{I})$ coincide if and only if $A B C$ is isosceles. A more geometric proof is given in [21, Section 11, problem 4, pp. 142-144]. Also, a generalization to higher dimensional orthocentric simplices is given in [18].

(v) The lines $L(\mathcal{G}, \mathcal{O}), L(\mathcal{G}, \mathcal{I})$, and $L(\mathcal{O}, \mathcal{I})$, together with several centers that they contain and the relative locations of these centers, are shown in Fig. 2. This figure shows the similarity between the Euler line $L(\mathcal{G}, \mathcal{O})$ and its rival $L(\mathcal{G}, \mathcal{I})$ and locates them as two medians of a triangle two of whose vertices are the orthocenter $\mathcal{H}$ and the Nagel center $\mathcal{N}$. The third vertex is labelled $\mathcal{X}$ and it does not seem to be a known center. This configuration points to two more centers, other than $\mathcal{X}$, that are denoted by $\mathcal{Y}$ (the midpoint of $\mathcal{H} \mathcal{N}$ ) and $\mathcal{Z}$ (the midpoint of $\mathcal{O I}$ ). We wonder whether these centers as well as the many lines that appear in this configuration are not already catalogued in [22].

(vi) According to [23], the line $L(\mathcal{O}, \mathcal{I})$ passes through 68 centers that include the Bevan point $X_{40}$ and the points $X_{46}, X_{56}, X_{165}$, and $X_{35}$. The trilinear equation of $L(\mathcal{O}, \mathcal{I})$ is

$$
(\cos B-\cos C) \alpha+(\cos C-\cos A) \beta+(\cos A-\cos B) \gamma=0 .
$$

In view of the fact that $(\cos B-\cos C, \cos C-\cos A, \cos A-\cos B)$ are trilinear coordinates of $X_{109}, L(\mathcal{O}, \mathcal{I})$ is the central line that should be denoted by $L_{109}$. For more on central lines, see [27].

In the two recent papers [16] and [19], the line $L(\mathcal{O}, \mathcal{I})$ is called the $O I$-line, and some of its properties are explored. According to [16], the point $X_{57}$ also lies on the $O I$-line. It is also proved in [19, Lemma 2] that the orthocenter of the intouch triangle, or equivalently the orthocenter of the cevian triangle of the Gergonne point, lies on the $O I$-line. One wonders whether this center is already catalogued in [22] and [23].

Acknowledgements. The first-named author is supported by Grant 11/2005 from Yarmouk University and the second-named author is supported by Grant 108/2007 from University of Sofia. The authors are also grateful to Professors Sadi Abu-Saymeh and Horst Martini for fruitful discussions.

\section{References}

[1] Abu-Saymeh, S.; Hajja, M.: Triangle centers with linear intercepts and linear subangles. Forum Geom. 5 (2005), 33-36

[2] Abu-Saymeh, S.; Hajja, M.: Other Brocard-like points of the triangle. Forum Geom. 5 (2005), 65-74.

[3] Abu-Saymeh, S.; Hajja, M.: In search of more triangle centres. Internat. J. Math. Ed. Sci. Tech. 36 (2005), 889-912.

[4] Abu-Saymeh, S.; Hajja, M.: Coincidence of centers for scalene triangles. Forum Geom. 7 (2007), 137146.

[5] Andreescu, T.; Andreca, D.: Complex Numbers from A to ... Z. Birkhäuser, Boston 2006. 
[6] Boskoff, V.G.; Suceava, B.D.: When is Euler's line parallel to a side of a triangle? College Math. J. 35 (2004), 292-296.

[7] Bradley, C.J.: Challenges in Geometry. Oxford University Press, New York 2005.

[8] Butchart, J.H.: Problem E411. Amer. Math. Monthly 47 (1940), 175; solution, ibid 47 (1940), 708-709.

[9] Carr, G.S.: Formulas and Theorems in Pure Mathematics. Chelsea Publishing Co., N.Y. 1970.

[10] Chao, W.W.: Problem 1506. Math. Mag. 69 (1996), 304; solution, ibid 70 (1997), 302.

[11] Charosh, M.: Problem E259. Amer. Math. Monthly 44 (1937), 104; solution, ibid 44 (1937), 541.

[12] Clarke, W.B.: Problem 199. National Math. Mag. 12 (1938), 410-411.

[13] Clement, P.E.: The concurrence of perpendiculars. Amer. Math. Monthly 65 (1958), 601-605.

[14] Conrad, S.: Problem E2501. Amer. Math. Monthly 81 (1974), 1026; solution, ibid 82 (1975), 939-940.

[15] Coxeter, H.S.M.: Introduction to Geometry. John Wiley, N.Y. 1969.

[16] Danneels, E.: The intouch triangle and the OI-line. Forum Geom. 4 (2004), 125-134.

[17] Dirnböck, H.; Schoißengeier, J.: Curves related to triangles: The Balaton-Curves. J. Geom. Graph. 7 (2003), 23-39.

[18] Edmonds, A.L.; Hajja, M.; Martini, H.: Orthocentric simplices for which the incenter lies on the Euler line. Submitted.

[19] Emelyanov, L.: On the intercepts of the OI-line. Forum Geom. 4 (2004), 81-84.

[20] Honsberger, R.: Mathematical Morsels. Dolciani Math. Expositions No. 3, MAA, Washington, D.C. 1978.

[21] Honsberger, R.: Mathematical Delights. Dolciani Math. Expositions No. 28, MAA, Washington, D.C. 2004.

[22] Kimberling, C.: Triangle centers and central triangles. Congr. Numer. 129 (1998), 1-285.

[23] Kimberling, C.: Encyclopedia of Triangle Centers. http://faculty.evansville.edu/ck6/encyclopedia/

[24] Longuet-Higgins, M.S.: On the ratio of the inradius to the circumradius of a triangle. Math. Gaz. 87 (2003), 119-120.

[25] Luenberger, F.: Problem E2002. Amer. Math. Monthly 74 (1967), 720, 860; solution, ibid 75 (1968), 909-910.

[26] Sivaramakrishnan, R.: Problem 758. Math. Mag. 43 (1970), 103; solution, ibid 43 (1970), 285-286.

[27] Weisstein, E.W.: Central line, from MathWorld - A Wolfram Web Resource. http://mathworld.wolfram.com/CentralLine.html

[28] Yiu, P.: Advanced Euclidean Geometry, 1992. http://www.math.fau.edu/Yiu/Geometry.html

\section{Mowaffaq Hajja}

Mathematics Department

Yarmouk University

Irbid, Jordan

e-mail: mowhajja@yahoo.com, mhajja@yu.edu.jo

Margarita Spirova

Faculty of Mathematics and Informatics

University of Sofia

Sofia, Bulgaria

e-mail: spirova@fmi.uni-sofia.bg 